OAI-PMH: http://www.indteca.com/ojs/index.php/Revista Scientific/oai

Artículo Original / Original Article

\title{
Rol del Gerente Educativo y su Interacción Comunitaria: Una Perspectiva Sociocrítica
}

\author{
Autor: Ciro Alberto Palacio Useche \\ Universidad Bicentenaria de Aragua, UBA \\ ciropalacio@hotmail.com \\ Turmero, Venezuela \\ http://orcid.org/0000-0002-5926-599X
}

Resumen

En el actual momento histórico de transformación educativa que vive el mundo, se requiere reafirmar y consolidar el trabajo conjunto del binomio escuela-comunidad. El presente estudio tiene como objetivo generar la aproximación teórica del rol del gerente educativo y su interacción con la comunidad, bajo el enfoque socio crítico, así como también en su entorno, representado en este caso, por los Consejos Comunales. Dichos Consejos Comunales como instancia de participación protagónica de los ciudadanos y ciudadanas en las acciones de la cotidianidad del quehacer social, se han convertido en una herramienta técnica, operativa y funcional que viabiliza la vida comunitaria en sana paz. La investigación fue desarrollada bajo el enfoque cualitativo - interpretativo, a través del método fenomenológico hermenéutico, lo que permitió develar, conocer, interpretar comprender y construir la aproximación teórica desde el conocimiento empírico y epistémico de los informantes clave. Las técnicas de análisis para el procesamiento de la información fueron el análisis categorial, la estructuración, la contrastación, la triangulación y la teorización. Los resultados obtenidos permitieron conocer las debilidades sobre la interacción de la gerencia con los integrantes de comunidad, así como el desconocimiento en el contexto jurídico y alcance en las políticas públicas y sociales, denotándose una separación que perjudica la formación integral de los estudiantes. Por tanto, se recomienda profundizar el trabajo aquí planteada a fin de permitir la práctica de estrategias y acciones pedagógicas que pudieran beneficiar en ambos sentidos.

Palabras clave: gerente; desarrollo comunitario; interacción social. 


\title{
Role of the Educational Manager and its Community Interaction: A Sociocrítica Perspective
}

\begin{abstract}
In the current historical moment of educational transformation that the world is experiencing, it is necessary to reaffirm and consolidate the joint work of the school-community binomial. The objective of this study is to generate a theoretical approach to the role of the educational manager and their interaction with the community, under the socio-critical approach, as well as in their environment, represented in this case, by the Communal Councils. These Communal Councils, as an instance of protagonist participation of citizens in the daily actions of social work, have become a technical, operative and functional tool that enables community life in a healthy peace. The research was developed under the qualitative - interpretative approach, through the phenomenological - hermeneutic method, which allowed to unveil, to know, to interpret understand and to construct the theoretical approach from the empirical and epistemic knowledge of the key informants. The analysis techniques for information processing were categorical analysis, structuring, contrast, triangulation and theorization. The results obtained allowed to know the weaknesses about the interaction of the management with the members of the community, as well as the ignorance in the legal context and scope in the public and social policies, denoting a separation that harms the integral formation of the students. Therefore, it is recommended to deepen the work proposed here in order to allow the practice of strategies and pedagogical actions that could benefit both ways.
\end{abstract}

Keywords: manager; community development; social interaction. 


\section{Introducción}

La dinámica actual en el planeta, se encuentra inmersa en un proceso de socialización y de competitividad, por tanto, en las organizaciones que luchan por ser más eficientes y productivas, se comienzan a generar constantes cambios que han ido apareciendo desde las últimas décadas del siglo pasado, bajo una perspectiva de desafíos en la gerencia educativa y su relación con el ámbito político y social en el siglo XXI. Para Valencia (2006), expone que:

Se vive en un mundo de cambio rápido y acelerado que tiene lugar en muchas áreas, abarcando aspectos económicos, políticos, sociales, científicos, tecnológicos, culturales y de comunicaciones, en los cuales los seres humanos y las organizaciones juegan un papel fundamental, pues se nace, se vive, se educa y se trabaja a su interior (pág. 1).

Interpretando a la autora, se necesita de un sistema educativo que abarque diferentes áreas, específicamente, lo social, económico, político, científico, tecnológico, entre otros, en la búsqueda de consolidar a un individuo pleno. Por lo tanto, amerita de nuevas demandas y cambios organizacionales. También, las instituciones educativas, las cuales deben renovar sus currículos especialmente en los niveles de Primaria y Media General para poder lograr una formación integral del individuo que necesita una sociedad altamente tecnificada y plena de adelantos e innovaciones tecnológicas, es decir, un currículum centrado en valores. Según Castro, Miquilena y Peley (2006), que señala:

Dentro de la cultura escolar, el modo como el liderazgo es conceptualizado y percibido por las organizaciones educativas es un factor clave para influir en la capacidad para hacer frente al cambio. Entendido en un sentido transformador encuentran como relevante; establecer una visión clara de la escuela, valorar y utilizar tareas de modo pertinente, entender el liderazgo como función que puede ser ejercida por cualquier miembro (pág. 9). 
Una institución es fundamental para el desarrollo normal de las actividades escolares es la comunidad educativa, la cual está integrada por los directivos, docentes, estudiantes, padres y/o representantes y personas proactivas del entorno local. A tal efecto, el personal directivo como gerente resulta altamente comprometidos para motivar al equipo de trabajo hacia una interacción comunitaria. Al respecto, Ander (1986), citado por Terry (2012), expone que la comunidad:

Sirve para designar a una agrupación organizada de personas que se perciben como unidad social cuyos miembros participan de algún rasgo, interés, elemento, objetivo o función común, con conciencia de pertenencia, situados en una determinada área geográfica en la cual la pluralidad de personas interacciona más intensamente entre sí que en otro contexto (pág. 4).

Interpretando al autor, la comunidad representa una organización de individuos que se interacción en un medio social, en la cual sus integrantes interacción con propósitos comunes, con sentido de pertenencia con el entorno donde se encuentra, significa, que se involucran todas las instituciones que encuentren cercana a ella. Por lo tanto, pertenecer a una comunidad se necesita que el individuo aprenda a convivir en otro ambiente fuera de la familia, a ser conscientes de sus deberes, responsabilidades, del rol que le corresponde desempeñar para analizar sus problemas y sus posibles soluciones al usar los recursos materiales y humanos existentes para organizar mejor su consumo y propiciar una vida familiar más feliz.

Las instituciones educativas como centro del quehacer comunitario, representan el papel principal como agente de cambio social. El Artículo 102 de la Constitución de la República Bolivariana de Venezuela (1999): refleja "La educación es un derecho humano y un deber social fundamental, es democrática, gratuita y obligatoria..." (pág. 16); por ello debe velar por todos los ciudadanos, involucrándose en su desarrollo, cuestión que puede lograrse 
a través de un proceso que busque la integración de todos los integrantes de la comunidad organizada. Este acercamiento le permitirá conocer el sistema de creencias, modos de vida y costumbres, contrarrestando así los antivalores $y$ fomentando la convivencia en sociedad.

Del mismo modo, la vida en comunidad se hace más factible el funcionamiento de todas las instituciones tanto públicas como privadas ofreciendo mutuamente, entre otros: servicios públicos, salud y asistencia de primeros auxilios; servicios municipales, de protección y seguridad; centros culturales, religiosos, recreativos y asociaciones profesionales y gremiales. Pero sobre todas las cosas se espera alcanzar un trabajo compartido entre integrantes de la escuela y la comunidad, para analizar, estudiar, abordar problemas y buscar vías de solución que satisfagan en ambos sentidos. Asimismo, el personal directivo y en especial el director, están llamados a su compromiso de trabajo comunitario ajustándose al momento histórico que vive el país, consustanciado con la formación social y política.

Por ello, Delgado (2011): señala "donde un individuo logre formarse para ser capaz de desarrollar lo verdaderamente humano y comprender las autonomías individuales, la participación comunitaria e internalizar la necesidad de congregarse para vivir en interacción con todo el entorno al que está expuesto" (pág. 45); así como sus habilidades y utilización de nuevas tecnologías para que todo docente como persona y profesional de la docencia necesita de un contexto social y político que los adueñe e identifique con la sociedad como eje integrador. Es muy importante decir que la educación está expuesta a cambios educativos donde el principal fin es, generar una educación social comunitaria en el cual la comunidad sea el principal promotor de cambios que beneficien a una educación de avance. Por lo tanto, todo docente es promotor social que no está aislado, sino vinculado estratégicamente en el plantel y la localidad.

No obstante, diversos autores, han planteado que hay evidencia que 
ello no siempre se cumple, debido, fundamentalmente a diferencias en los marcos referenciales con los que, tanto profesores como directivos, abordan para desarrollar la interacción social en las escuelas. Entre ellos, Romero (2006), indica: "en el vínculo pedagógico y directivos existen entre familia y escuela, observamos decrecimiento participación de los padres en reuniones, actos escolares, fiestas recreativas y citaciones de los docentes" (pág. 134). Esto evidencia el desarrollo de una praxis docente con gran desarrollo a nivel académico sólo dentro de la escuela, sin embargo, con problemas de corresponsabilidad y conciliación a los diferentes entornos sociales, implicando, visiblemente, un recorrido entre él y lo que incumbe a la visión actual de un mundo compartido.

Por su parte, Gomero (2007): hace referencia que a través de un “...estudio de los procesos comunicativos desarrollado en una institución escolar... se ha demostrado la importancia de las relaciones que se establecen entre las escuelas y el entorno social" (pág. 23), por cuanto, el plantel no es una institución capaz de actuar en forma parte en la formación de niñas y niños. De acuerdo con Creel (2001): es de resaltar que la escuela “...forma parte de una red de instituciones que en mayor o menor medida están implicadas en la educación" (pág. 89). Es por ello, que se considera a la educación es considerada el medio en la formación, en la cual los individuos se establecen en personas sociales, donde se obtienen las características indispensables para comprender el ámbito social.

En consecuencia, la investigación se planteó como objetivo generar la aproximación teórica del rol del gerente educativo y su interacción con la comunidad, bajo el enfoque socio crítico. Es decir, se buscan las transformaciones sociales que ocurren en las instituciones educativas y el acercamiento hacia a ellas. Igualmente, profundizar en el análisis crítico, Interpretativo y hermenéutico del rol que asumen los gerentes frente a los problemas o situaciones de los problemas que en encuentran en las 
comunidades, con énfasis en la autorreflexión, el humanismo y valoración de los individuos, en el seno en cual ocurre la realidad del colectivo.

La integración educativa está orientada hacia la colaboración de representantes, docente, directivos y localidad en general, en la búsqueda de una unión fundamental en pro del desarrollo sociocultural educativo, donde el hombre enfoque las ideas y participe de manera espontánea. El gerente educativo se caracteriza por ser un servidor público, y está llamado a propiciar un entorno adecuado para que se consolide la interacción comunitaria. Desde este punto de vista, el presente antecedente muestra la necesidad en los procesos de cambio del entorno escolar para alcanzar los verdaderos objetivos que rigen la educación integral de los estudiantes.

En la actualidad, lo que se busca es crear un seguimiento de las estrategias dando inicio a la integración educativa, fortaleciendo los lazos en la escuela y la comunidad, las cuales deben ser apoyadas y respaldadas por el personal del recinto educativo. Asimismo, fomentar la disposición educativa en cualquier ambiente social conformando así cada una de las familias que conforman la localidad como miembros de rescates educativos y valores que ayuden al aprendiz a crear, una conducta sana del buen vivir.

Todo lo anterior permite precisar, que las teorías relacionadas con el presente estudio tienen estrecha relación en enfatizar la importancia del Gerente Educativo y su interacción comunitaria. Asimismo, resaltar las diferentes situaciones que inciden a favor de la distribución y cumplimiento de acciones dirigidas hacia el alcance de estrategias y metas planteadas por las instituciones educativas.

Por lo tanto, la interacción de actividades afiliadas en las instituciones escolares y las localidades, en la cual los estudios que se han realizado sobre la temática, destacan la relevancia e importancia del protagonismo que tiene las funciones del educador en el rol de actor y gestor en el desarrollo $d$ convivencias, prácticas y experiencias en la interacción permanente en el 
proceso enseñanza-aprendizaje. Finalmente, se confirma la importancia de la acción comunitaria y la imperiosa necesidad de impulsar el rol del gerente como productor de ideas, conocimientos, innovaciones y propuestas; así como también la actuación en el desarrollo tareas, programas y proyectos que accedan al acercamiento con los miembros de la comunidad. Lo antes expuesto, requiere se actitudes proactivas, comprometidas, corresponsables con cada uno de los integrantes educativos y comunitarios en función de consolidar la misión, visión y valores de la institución y el entono en el cual se encuentra.

Tal como lo refiere Ruiz (2010a): el aprendizaje como proceso universal "es como el hilo conductor de las organizaciones inteligentes, por tal razón, mediante una educación participativa que facilitaría los procesos que dan como resultados aprender juntos a compartir responsabilidades efectivamente de los factores y los resultados" (pág. 56). Se desarrollan a imágenes y supuestos efectivos en atención a los requerimientos de las organizaciones. Además, las organizaciones inteligentes permiten tener una visión compartida que conllevan a un cambio de comportamiento gerencial, al interrelacionarse las experiencias del personal adscrito en este programa con la experiencia del gerente.

Este enfoque antes mencionado, se puede aplicar a la Educación demostrando así que el gerente deja de ser clásico para ejercer una función de diseñador, administrador, organizador, y planificador; convirtiéndose en un ser activo que interactúa con los medios y el recurso a fin de realizar la toma de decisiones y asumir la responsabilidad de ejecutar los procesos administrativos. De igual manera, Ruiz (2010b), expone:

En el campo educativo, la gerencia es de interpretación conceptual relativamente, por cuanto las organizaciones dedicadas a la enseñanza tienen un fin social, por lo cual en el contexto de su evolución se ha aplicado predominantemente el aspecto administrativo en función de aplicar una efectiva 
planeación, que también se desarrollan mediante las funciones de planeación, distribución, dirección y control, pero no con un fin o beneficio económico, sino que procura el fomento del talento humano, según los planteamientos de un proyecto educativo nacional (pág. 56).

En consecuencia, el proceso gerencial, en el campo educativo constituye un proceso fundamental en la evaluación de las organizaciones; ya que debe existir un buen gerente que maneje y ejecute la planificación, la organización, el dirigir y controlar la misma procurando el desarrollo del talento humano, materiales y financieros según un proyecto educativo nacional.

En una organización siempre es indispensable una efectiva gerencia, por lo tanto, es necesario efectuar dos tipos preguntas primordiales: ¿Por qué y cuándo la gerencia es necesaria? De ahí, la respuesta estará en función al origen propio de la gerencia en la cual es garante del éxito o fracaso de una institución. La aseveración de que la gerencia es la responsable del triunfo o fracaso, quiere decir, que se amerita de una gerencia más no indica cuando ella es requerida.

En consecuencia, en toda organización se amerita de un conjunto de personas que busquen objetivos comunes, por lo tanto, se requiere de trabajar en colectivo y unir esfuerzos para alcanzar los propósitos, metas y estrategias en la productividad y bienestar en la misma. Los miembros del equipo deben disciplinar, en determinado término, las aspiraciones individuales para lograr los fines de todos y el gerente debe proporcionar el liderazgo, coordinación y orientación guiadas a la acción grupal. Po lo tanto, en las instituciones, les incube mantener equipos de alto desempeño en consolidar efectiva comunicación y toma de decisiones.

En este orden de ideas, puede observarse que la actividad correctiva del control da lugar, de manera invariable, a una redefinición de los planes, de allí que muchos investigadores en el proceso gerencial piensan que la función 
de control-dirección son relevantes y son parte esencial de un ciclo continuo de planificación-control-planificación.

En consecuencia, el proceso gerencial puede precisarse como una serie de funciones separadas, sin embargo, cada una de ellas engranadas cabalmente en un comportamiento aparte. Pero no es así, aunque el proceso, para que pueda comprenderse, debe ser subdividido y cada aspecto analizado por separado. En la praxis el gerente efectúa con frecuencia o lo realiza paralelamente a las funciones de planeación, dirección, organización y control. Esto con la finalidad de mejorar la calidad y elevar la productividad en la misma.

Para Hernández (2000): plantea que "como hombre son muy identificables con los roles por el modo que cumple con las expectativas percibidas cualidades del Gerente Educativo" (pág. 29). El gerente podría desarrollar el rol del director al establecer objetivos y dirigir a sus subalternos en las responsabilidades necesarias para lograr los objetivos preestablecidos, así mismo actuar como coordinador de las acciones del trabajo del staff facilitando el trabajo de las partes integrantes para que cada uno pueda operar con la mayor eficiencia en función del logro de los objetivos organizacionales establecidos por el grupo.

Es decir, como líder orientado hacia el grupo con proposiciones relacionadas tanto con el método, como con la dirección para ser aprobados por el grupo, es de allí donde el gerente educativo capacita al staff para aprobar sus orientaciones teóricas y para desarrollar sus propias bases para la acción. Por otra parte, plantea que el trabajo y la posición del gerente educativo están relacionados al factor antecedente llamado entorno social. En los últimos años algunos autores, tales como Márquez (2000):

Han verificado la tesis de que existe discrepancia entre la naturaleza en las perspectivas del centro educativo como institución y el entorno de posibilidades en términos de: (a) la finalidad de la organización, (b) las acciones a ejecutarse; (c) los 
procesos adecuados y (d) el comportamiento del gerente como dirigente y promotor social (pág. 72).

Efectivamente, existe variedad de teorías sobre el liderazgo y el impacto dentro de las organizaciones. Actualmente las organizaciones modernas requieren gran coordinación de esfuerzos comunes, alta especialización y un liderazgo compartido. La Gerencia que puede generar cambios debería proveer las destrezas para implementar y mantener dichos cambios substanciales mientras prestan su utilidad a la organización y a las personas. La realidad no es unilateral sino multicausal y la manera de abordarla es a través de una visión integral.

En este caso el gerente educativo deberá conjugar sus esfuerzos, destrezas de la realidad de su entorno con una actitud positiva o negativa de lo que acontece. Al respecto, Martínez (2001a): afirma que "la actitud es una forma de respuesta, a alguien o a algo aprendida y relativamente permanente" (pág. 86). También, Martínez (2001b) expone "este término de actitud ha sido definido como una reacción afectiva positiva o negativa hacia un objeto 0 proposición abstracto o concreto denotado" (pág. 44). En otras palabras, las actitudes son estudiadas y el resultado pueden ser características de motivos biológicos y sociales, tales como: el hambre, la sed y el sexo que son aprendidas. Las actitudes suelen permanecer en el tiempo. Las mismas están encaminadas hacia un objeto o símbolo específico. Las actitudes pocas veces son individuales; por lo general son tomadas en equipo y con mayor armonía.

Por consiguiente, las actitudes se conciertan de tres (03) elementos: el pensamiento del individuo (relacionado a lo cognitivo), lo que concibe el sujeto (representa el aspecto emocional) y la propensión a declarar los pensamientos y sentimiento (relativo al componente conductual). Así pues, la teoría afirma que un líder debe presentar cualidades donde el sentido práctico de las acciones encaminadas hacia la consecución de los objetivos, es decir, tener 
capacidad para comunicar correctamente la misión, visión y objetivos de la compañía logrando la adhesión de las personas, de igual modo el líder debe transmitir confianza y credibilidad y con un conocimiento de sus propias habilidades y todo esto demuestra que el líder debe poseer cualidades para el control de la atención, del significado de confianza de el mismo como personal para demostrar su habilidades de gerente.

Del mismo modo, la actitud puede precisarse como una manera de motivación social (de carácter secundario) frente a la motivación biológica (de carácter primario), quien es la encargada de guiar y situar la acción en la conclusión de objetivos y fines. Las emociones se vinculan con las actitudes de un individuo frente a determinada situación. Se concibe por actitud una predisposición, habilidad o pensamiento de actuar en un contexto específico. Sin embargo, en la actitud se encuentran inmersos muchos elementos, en los cuales se potencian los pensamientos y sentimientos. Por ejemplo, en la indagación de una carrera profesional, si la actitud es propicia, en ella se hayan pensamientos positivos relativo a ella; así como emociones de apego y afecto por los estudios. Las mociones son elementos esenciales en las actitudes.

Es relevante acotar, que una vez formada la actitud no es fácil que pueda ser modificada, razón por la cual depende de las creencias, convivencias y valores de la familia de origen. Efectivamente, dichas actitudes pudieron crearse en los primeros años de vida del individuo y haberse fortalecido a largo de la vida. Además, otras actitudes son adquiridas en la sociedad, como es el caso de cualidades negativas de robo y de homicidio, y de último otras estriban directamente del sujeto. Sin embargo, existen actitudes que pueden cambiarse o modificarse.

En la interacción continua, se plantea la posibilidad de proteger la imagen, debido a que se accede a interactuar con una mejor fluidez y está imagen es muy importante, porque muchos gerentes se sirven de ella para actuar de forma adecuada frente a cualquier situación o conflicto. Es por lo que 
la gran mayoría de las interacciones se efectúan por medio de la utilización del lenguaje y la conversación. En toda interacción debe existir una efectiva comunicación, para que todos los integrantes de la organización mantengan relaciones armónicas, tranquilas y pacíficas en función de resguardar la imagen y por ende garantizar un ambiente en la cual se potencien las actitudes.

En el aspecto educativo y apoyado en el paradigma ecológico, enmarcado en el aspecto ambiental y a las disimiles interacciones en los seres vivos y los objetos que conforman el espacio cercano a ellos, los mimos representan las bases para la integración de la escuela con la existencia de la comunidad; sedimentando el modelo educativo en atención a consolidar el ambiente, la erosión, la destrucción de las especies por parte de parte de los seres humanos, la conservación y resguardo.

No obstante, la ecología humana no aleja al hombre de su ambiente, al contrario, ella comprende la actuación del hombre, tomando en cuenta todos los elementos que se encuentran en el contexto fuera y dentro de ella. Asimismo, involucra a todo el hombre con el ambiente, tal como está presentado, es decir, de manera holística. Esto significa, la forma como se interacciona para consolidar su hábitat, esto da lugar a la caracterización de la estructura de la comunidad, la cual está basada con sus rasgos externos y medibles.

En otro orden de ideas, Harolt (2012): plantea que la integración de padres y representantes, es "...la concurrencia de actores y acciones conducentes a garantizar el ejercicio de los derechos de los niños, las niñas y los adolescentes" (pág. 163); además la integración de los miembros de la comunidad escolar, es un hecho reconocido que se manifiesta en cualquier sociedad democrática, ya que la constitución y pervivencia de ésta se fundamenta en la colaboración de los ciudadanos y ciudadana en la construcción de equipos de trabajos fortalecidos. 
De allí que, la intervención de otros miembros de la comunidad educativa en los procesos de organización y gestión está justificada por las leyes (Normativo de Comunidad y Ley de Educación); la complejidad de la gestión reclama la presencia numerosa de personas en función de colaborar en el fortalecimiento de la integración escuela-comunidad, según sus capacidades y competencias. Al respecto, Martínez (2018), plantea que:

La participación comunitaria está vinculada a impulsar el desarrollo de una comunidad, buscando cambios efectivos en la evolución de la realidad en la cual se encuentra inmersa. Además, incidirá en la formación y el potencial en la autogestión de recursos que contribuyan con el bienestar social, educativo, ambiental y cultural de los individuos (pág. 293).

La autora refleja, que la participación comunitaria está directamente asignada a promover integración efectiva en el desarrollo de acciones que contribuyan a mejorar las deficiencias y motivaciones de los integrantes de la localidad, en la cual se les dé incursión a los integrantes de la escuela en función de elevar la educación, el ámbito social, político, económico y ambiental.

\section{Metodología}

La metodología utilizada fue focalizada en el enfoque cualitativo. La misma es abordada desde la intersubjetividad, por lo que se requirió de operaciones acordes a la naturaleza del fenómeno social estudiado. De allí, Ugas (2011): sostiene "La metodología actúa como una bisagra que relaciona método y epistemología" (pág. 17).

La posición asumida en el estudio, por parte del autor, fue el enfoque cualitativo, que permitió la integración del sujeto investigador y la producción de la postura en la creación del pensamiento y la obtención un conocimiento a profundidad de la realidad que le rodea, partiendo de la complejidad del 
pensamiento, bajo un discurso interpretativo que fue manejado en los consejos comunales, todo ello fundamentado en la hermenéutica que se describe de acuerdo a la intencionalidad del investigador en alcanzar la interpretación del conocimiento.

El método que se asumió para la realización del estudio fue el fenomenológico-hermenéutico, porque develó la realidad de los hechos. Se caracterizó por la construcción de un acercamiento teórico o comprensiones de una realidad aproximada. Esto quiere decir, que lo estudiado guardó una relación de correspondencia y pertinencia con las realidades; que no son propiamente reales ni tampoco puramente formales, sino que reveló la posición de las realidades que se viven en determinados momentos en una situación dada.

En este sentido, Sandín (2003): expresa "un estudio fenomenológico describe el significado de las experiencias vividas por una persona o grupo de personas acerca de un fenómeno" (pág. 151). Por lo tanto, la realidad surge no como un punto de vista objetivo, sino como una disposición de los significados que los sujetos dan a dicha realidad, desde cualquier escenario $o$ circunstancia sociopolítica en la cual esté inmerso el fenómeno. En cuanto a los informantes clave, según Robledo, citado por Mejías (2016), considera que:

Aquellas personas que por sus vivencias, capacidad de empatizar y relaciones que tienen en el campo pueden apadrinar al investigador convirtiéndose en una fuente importante de información a la vez que le va abriendo el acceso a otras personas y a nuevos escenarios (pág. 14).

Interpretando a la autora, los informantes clave son aquellos individuos con habilidades, destrezas, capacidad, potencialidad y relaciones pueden proporcionar información relevante con la temática que se esté estudiando. De allí, que los informantes, de la presente investigación estuvieron constituidos por dos (02) gerentes del Liceo Bolivariano "Colinas del Llano" y tres (03) 
integrantes del Consejo Comunal del Sector Araguaney de la comunidad de Ciudad Varyna, municipio Barinas del estado Barinas.

De acuerdo con la investigación, el paradigma, el enfoque y la metodología que se utilizó el investigador, se asumieron las técnicas para este estudio tomando en cuenta la naturaleza de esta, es así, que se partió de la observación participante. Al respecto, Taylor y Bogdan (1994), plantean: "involucra la interacción social entre el investigador y los informantes en el milieu (escenario social, ambiente o contexto) de los últimos, y durante la cual se recogen datos de modo sistemático y no intrusivo" (pág. 31). Igualmente, se busca la selección del escenario social, el acceso a dicho escenario, la interacción con los porteros que permiten el acceso del investigador al escenario.

Igualmente, se utilizó la entrevista en profundidad, la cual permitió que el investigador pudiera estudiar a los informantes clave en su comportamiento y la creación de conocimiento cognoscitivo. Al hacer referencia de la entrevista en profundidad, como técnica de recolección de información, se destaca, según Gómez (2006): que es "el encuentro cara a cara entre el investigador y los informantes clave" (pág. 62). Esta técnica permitió indagar las realidades de los informantes clave.

Por su parte, la Categorización, de acuerdo a la profundidad del tema en estudio, la cual es descrita por Martínez (2006a): "La categorización consiste en la segmentación en elementos singulares, o unidades, que resultan relevantes y significativas desde el punto de vista del interés del investigador y su equipo" (pág. 164); por ello, la categorización se realizó por unidades de registro, es decir, estableciendo una unidad de sentido haciendo posible la creación de otra, dentro de un contexto teórico registrado.

Del mismo modo, la triangulación, la cual tuvo el propósito de generar mayor soporte teórico a la comprensión teórica estipulada para el estudio, se partió de la triangulación de información o datos, a la cual Martínez (2006b): 
manifiesta que "se utiliza una amplia variedad de datos para realizar el estudio que proviene de diversas fuentes de información" (pág. 178). De esta se desprende que, es a partir de la información recolectada por el autor del estudio, se realizó la triangulación de las opiniones de los informantes clave.

\section{Análisis e Interpretación de la Realidad}

El análisis e interpretación de la realidad fue caracterizada por la heurística por su nivel de abstracción, en donde se descubrieron o develaron nuevos elementos, extraídos de los códigos axiales de los relatos de los informantes clave, extracción que fue sometida a un proceso dialéctico (tesisantítesis-síntesis), lo que permitió una nueva forma de ver las cosas, estas nuevas cosas, es lo que se llama categorías y subcategorías emergentes, es decir que subyacieron a la postre de la persovisión de los informantes clave.

De allí que, con la aplicación de la categorización, la cual fue obtenida con las expresiones verbales de los informantes clave, tal como se evidencia en las expresiones de algunos de ellos: Informante número 1: Los gerentes no han podido consolidar una buena interacción con los miembros del Concejo Comunal, es posible, por un lado, la diversidad de pensamiento filosófico político, y por otro que los canales comunicacionales parecen ser los menos adecuados. El informante número 2: Los directivos carecen de un rol gerencial efectivo que garantice la participación a los miembros del Consejo Comunal a la problemática que padece la institución.

La información suministrada por lo informantes fue triangulada, obteniendo que los entrevistados coincidieran en fortalecer el rol gerencial del directivo en la interacción con los integrantes del Consejo Comunal y la consolidación de canales eficientes de comunicación. Además, gestionar talleres de formación, en cual se garantice el conocimiento de las funciones que tienen los Consejo Comunales y la participación en la elaboración de planes, programas y proyectos en el ámbito educativo. 
Al mismo tiempo, los hallazgos emergentes más significados estuvieron centrados en: la herramienta social para la gestión inteligente, representaciones sociales en la cotidianidad educativa-social y cosmovisión de las funciones gerenciales del gerente. Con estos hallazgos se perfiló en generar la aproximación teórica del rol del gerente educativo y su interacción con la comunidad, en el entorno de los Consejos Comunales. En consecuencia, se perfila innovar y modernizar a los directivos en ámbitos más sinérgicos, proactivos y de comunicaciones efectivas.

\section{Conclusiones}

Existe una deficiente comunicación entre los miembros del consejo comunal y los gerentes educativos adscritos al plantel. Sin embargo, según los resultados del diagnóstico, se pudo constatar que cada uno de los actores (miembros de la escuela y de los consejos comunales), trabajan de manera individual por el bienestar común.

Por otra parte, se evidenció que cada grupo en estudio se interrelacionan con organismos competentes para la solución de problemas del entorno y sea educativo o comunitario. Lo cual representaría una fortaleza aprovechable si él se conformará equipos de trabajos entre ambos sectores. Aunque existe disposición al trabajo mancomunado por parte de los directivos y los representantes de los consejos comunales no se ha concretado la interacción y el trabajo en equipo en ambos sentidos.

Es importante destacar, que el desconocimiento de las funciones y el perfil de los gerentes educativos por parte de los consejos comunales, ha traído como consecuencia juicios a priori por parte de los mismos, acerca del desempeño de los directivos. Este elemento limita las buenas relaciones entre las poblaciones en estudio. Así pues, resulta oportuno señalar que el liderazgo que se ejerce en la institución educativa es de carácter democrático y con responsabilidades compartidas. 


\section{Referencias}

Ander, E. (1986). Diccionario del trabajo social. 8ª edición. México: Editorial Ateneo, pág. 83.

Castro, E., Miquilena, E., \& Peley, R. (2006). Las Nuevas Tendencias del Liderazgo: Hacia una Nueva Visión de las Organizaciones Educativas. Omnia, 12(1), 83-96, ISSN: 1315-8856. Recuperado de: http://www.redalyc.org/pdf/737/73712104.pdf

Constitución de la República Bolivariana de Venezuela (1999). Gaceta Oficial Extraordinaria № $36.860,30$ de diciembre. Caracas, Venezuela: Asamblea Nacional.

Creel, J. (2001). La Escuela. México: Humanitas.

Delgado, L. (2011). Formación del Individuo. 3ra. Edición. Madrid, España: McGraw-Hill.

Gomero, I. (2007). Procesos Comunicacionales. Colombia: Norma.

Gómez, R. (2006). Los Desafíos Actuales Metodológicos. España: Romor. Harolt, P. (2012). Integración Educativa. Madrid, España: Océano.

Hernández, R. (2000). Gerente Educativo. Caracas, Venezuela: Fondo Editorial de la Universidad Pedagógica Experimental Libertador, FEDUPEL.

Márquez, L. (2000). Educación y Formación. Colombia: Trillas.

Martínez, C. (2018). Participación Comunitaria Orientada hacia la Valoración del Patrimonio Cultural. Revista Scientific, 3(8), 290-300. Recuperado de:

https://doi.org/10.29394/Scientific.issn.2542-2987.2018.3.8.15.290-300 Martínez, M. (2006a,b). Ciencia y Arte en la Metodología Cualitativa. México: Trillas.

Martínez, T. (2001a,b). Actitud. Buenos Aires, Argentina: Humanitas.

Mejías, A. (2016). Articulación en la Promoción Ambiental desde la Tríada:

Escuela, Familia, Comunidad. Revista Scientific, 1(1), 8-19. 
Recuperado de:

https://doi.org/10.29394/scientific.issn.2542-2987.2016.1.1.1.8-19

Romero, E. (2006). Pedagogía y Gerencia. España: Espasa.

Ruiz, L. (2010a, b). La Gerencia. México: McGraw-Hill.

Sandín, M. (2003). Investigación Cualitativa en Educación: Fundamentos

y Tradiciones. España: McGraw-Hill.

Taylor, S., \& Bodgan, R. (1994). Introducción a los Métodos Cualitativos de Investigación. La Búsqueda de Significados. ISBN: 84-7509-816-9. Barcelona, España: Ediciones Paidós. Recuperado de: https://iessb.files.wordpress.com/2015/07/05 taylor mc3a9todos.pdf

Terry, J. (2012). Aproximación al Concepto de Comunidad como una Respuesta a los Problemas del Desarrollo Rural en América Latina. CCCSS, Contribuciones a las Ciencias Sociales, (12), ISSN: 19887833. Recuperado de:

http://www.eumed.net/rev/cccss/22/concepto comunidad.html

Ugas, G. (2011). La Articulación Método, Metodología y Epistemología. Taller Permanente de Estudios Epistemológicos en Ciencias Sociales. México: Ediciones del TAPECS.

Valencia, P. (2006). Desarrollo Organizacional Aplicado a la Educación Superior. Modelo de Análisis. REPES, Revista Electrónica de Educación y Psicología, 2(4), 1-8, ISSN: 1692-5637. Recuperado de: http://revistas.utp.edu.co/index.php/repes/article/view/5257 


\section{Ciro Alberto Palacio Useche}

e-mail: ciropalacio@hotmail.com

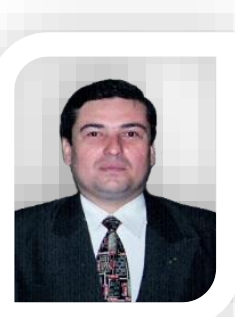

Nacido en Táriba, estado Táchira, San Cristóbal, Venezuela. Licenciado en Educación Mención Ciencias Sociales: Especialidad Historia y Geografía, Universidad Católica del Táchira; Magister Scientiarum en Gerencia y Liderazgo en Educación en la Universidad Fermín Toro, oficina de Enlace Barias; Abogado en la Universidad Bolivariana de Venezuela, Barinas; Profesor de pregrado y postgrado en las áreas de: Turismo, Lenguaje y Comunicación, Metodología y Proyectos de Investigación; Instructor Colaborador del INCES en Seminarios de Relaciones Públicas, Toma de Decisiones San Cristóbal, Mérida y Guasdualito, Geografía e Historia de Venezuela, Historia de la Guardia Nacional Bolivariana, Comando Zona Nº 33 en la UNELLEZ, Barinas en el año 2003; Coordinador Local en Universidad Bicentenario de Aragua.

El contenido de este manuscrito se difunde bajo una Licencia de Creative Commons ReconocimientoNoComercial-Compartirlgual 4.0 Internacional 